\title{
Radiometric Calibration of Compact Infrared Camera (CIRC) for Earth Observation
}

\author{
Eri Kato*, Haruyoshi Katayama, Masataka Naitoh, Masatomo Harada, \\ Ryoko Nakamura, Michito Sakai, Yasuhiro Nakajima, \\ Koji Nakau', Yoshio Tange and Ryota Sato \\ Satellite Applications Mission Directorate I, Japan Aerospace Exploration Agency, \\ 2-1-1 Sengen, Tsukuba, Ibaraki 305-8505, Japan \\ ${ }^{1}$ Graduate School of Science, Hokkaido University, \\ Kita 8, Nishi 5, Kita-ku, Sapporo 060-0808, Japan
}

(Received December 2, 2013; accepted February 6, 2014)

Key words: remote sensing, uncooled infrared detector, wildfire, ALOS-2, JEM-CALET

We have developed a compact infrared camera (CIRC) with an uncooled infrared array detector (microbolometer) for space application. CIRC is the first microbolometer sensor without a calibration function in orbit, like a shutter system or an onboard blackbody. The main objective of the CIRC is the technology demonstration of wildfire detection. Microbolometers are suitable for application in resource-limited sensor systems or small satellites because no cooling systems are required. Another main characteristic of the CIRC is its use of athermal optics. The athermal optics system compensates for defocus occurring owing to temperature changes. The CIRC achieves a small size (approximately $200 \mathrm{~mm}$ ), light mass (approximately $3 \mathrm{~kg}$ ), and low electrical power consumption $(<20 \mathrm{~W})$ by employing athermal optics and a shutterless system. The shutterless system suggests the need to devise a method of achieving stray-light correction and temperature calibration without the use of a mechanical shutter for calibration. We completed the ground calibration test of the CIRC Proto Flight Model (PFM) in a vacuum environment and at ambient temperature ranging from -15 to $50{ }^{\circ} \mathrm{C}$ using blackbody images at various temperatures $\left(-30\right.$ to $\left.50^{\circ} \mathrm{C}\right)$, and accomplished the method of temperature correction to achieve temperature accuracy of $4 \mathrm{~K}$ without a calibration function. In this paper, we report on the constructed radiometric algorithm and results relevant to imaging and radiometric quality.

${ }^{*}$ Corresponding author: e-mail: katoh.eri@jaxa.jp 


\section{Introduction}

Microbolometers are widely used in commercial and military applications. In the light of recent progress in micro-electromechanical systems (MEMS) technology, highresolution, large-format microbolometers are being developed by many companies. These devices possess the advantage that they do not require a cooling system such as a mechanical cooler. Sensors without a cooling system for a detector are small in size, have light mass, and consume less power. Although the sensitivity of a microbolometer is less than that of $\mathrm{HgCdTe}$-based photonic infrared detectors, its advantage of not requiring a cooling mechanism makes it suitable for use in small satellites or sensor systems whose resources are limited. For this reason, microbolometers begin to be applied for some space applications. In planetary missions, the Longwave IR (LIR) camera onboard the Venus Climate Orbiter (PLANET-C), ${ }^{(1)}$ which was launched in 2010, also uses a microbolometer array. In earth-observation missions, the Infrared Spectral Imaging Radiometer (ISIR)(2) experiment of NASA/GSFC tests the potential of a microbolometer for application to thermal infrared imaging. The ISIR was flown as a low-cost shuttle experiment on the STS-85 space shuttle mission in 1997. Recent missions to measure the atmosphere vertical profiles such as $\mathrm{CALIPSO}^{(3)}$ and EarthCARE ${ }^{(4)}$ also use a microbolometer as an imaging infrared instrument. JAXA began a research for the application of a microbolometer to Earth observation since 2000.

To demonstrate microbolometer technology to thermal infrared imaging from space, we have developed the compact infrared camera (CIRC). ${ }^{(5-7)}$ CIRC does not have a calibration function in orbit such as a shutter system or an onboard blackbody. The main objective of the CIRC is to detect wildfires, which are a major and chronic disaster that affects many countries, particularly those in the Asia-Pacific region. There are research studies suggesting that global warming and climate change may accelerate the severity of wildfires and other natural disasters. In the Sentinel Asia project, which will share disaster information in near real time across the Asia-Pacific region, wildfire has been chosen as one of the important phenomena to be monitored. Therefore, their early detection is important. An effective means of early detection is to increase their observation frequency. Our aim is to realize frequent observations by loading CIRCs in as many satellites as possible by exploiting their small size, light mass, and low power consumption. Other mission targets of the CIRC include the observation of volcanoes and urban heat islands. The CIRC will be carried as a technology demonstration payload on board the Advanced Land Observing Satellite-2 (ALOS-2) ( $^{(8)}$ and the CALorimetric Electron Telescope (CALET) ${ }^{(9)}$ which will be attached to the Japanese Experiment Module (JEM-EF) at the International Space Station (ISS).

In this paper, we present the verification results of the athermal characteristics and the calibration without a calibration function using the CIRC Proto Flight Model (PFM). In $\S 2$, we provide an overview of the ALOS-2 and CALET. The specifications of the CIRC are described in $\S 3$. In $\S 4$, data correction algorithm and the ground calibration results of the CIRC PFM on board the ALOS-2 and CALET are presented. Airborne observations made using the CIRC ground test model are presented in $\S 5$. In $\S 6$, we conclude the paper. 


\section{ALOS-2 and CALET}

The ALOS-2 is a follow-on satellite of the ALOS, which has been contributing to cartography, regional observation, disaster monitoring, and resource surveys since its launch in 2006. JAXA has been conducting research and development activities to improve the wide-field and high-resolution observation technologies developed for ALOS to further address social needs. These social needs include (1) disaster monitoring of damage areas, both in considerable detail and over large geographies; (2) continuous updating of data archives related to national land and infrastructure information; (3) effective monitoring of cultivated areas; and (4) global monitoring of tropical rainforests to identify carbon sinks. The ALOS-2 will be launched in JFY 2013. The ALOS2 inherits the L-band synthetic aperture radar (SAR) observations from the ALOS PALSAR with enhanced capabilities. Table 1 lists the baseline specifications of the ALOS-2. The mounting location of the CIRC is shown in Fig. 1. In essence, the CIRC will take images of the target area when the SAR is pointing to the right at an off-nadir angle of $30^{\circ}$.

The CALET will be mounted to a facility on Japan's Kibo laboratory aboard the ISS to observe high-energy cosmic rays. The CALET is expected to reveal various mysteries of the universe such as: (1) the nature of the sources of high-energy particles and photons through the high-energy spectrum, (2) the details of particle transportation in the galaxy, and (3) signatures of dark matter in either high-energy electrons or the gamma ray spectrum. It will also be capable of monitoring gamma ray transients and solar modulation. The CALET will be launched in JFY 2014. The baseline specifications of the CALET are listed in Table 1. The CIRC will be mounted on the side panel of the CALET, as shown in Fig. 1.

\section{CIRC}

The main objective of the CIRC is to demonstrate the technology for detecting wildfires using a microbolometer. The CIRC includes athermal optical and shutterless systems. The athermal optical system of the CIRC was designed by combining two infrared materials: germanium and chalcogenide. The camera's temperature does not need to be controlled using a heater. Thus, we realized a CIRC with a small

Table 1

Baseline specifications of ALOS-2 and CALET.

\begin{tabular}{lcc}
\hline Satellite & ALOS-2 & CALET \\
\hline Size & $9.9 \times 16.5 \times 3.4 \mathrm{~m}^{3}$ & $1.0 \times 0.8 \times 1.85 \mathrm{~m}^{3}$ \\
Mass & $<2000 \mathrm{~kg}$ & $<0500 \mathrm{~kg}$ \\
Bus power & $>5200 \mathrm{~W}$ & $>500 \mathrm{~W}$ \\
Communication & $<800 \mathrm{Mbps}$ & $<300 \mathrm{kbps}$ \\
Orbit & $628 \mathrm{~km}$ & $407 \mathrm{~km}$ (nominal) \\
\hline
\end{tabular}




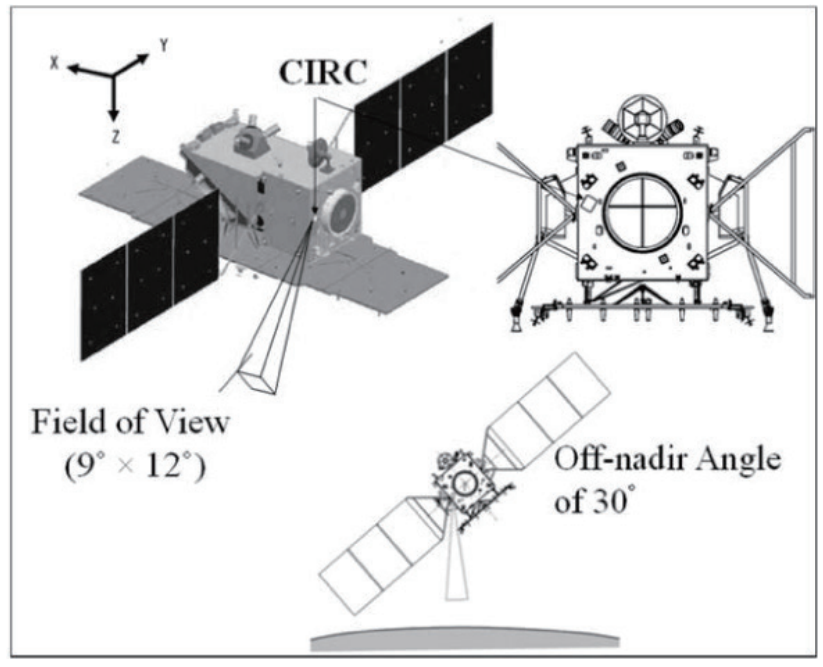

(a)

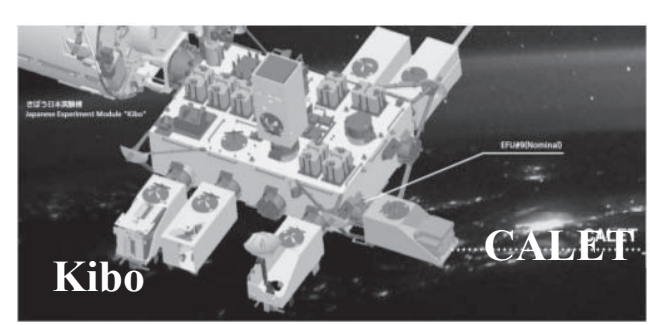

(b)

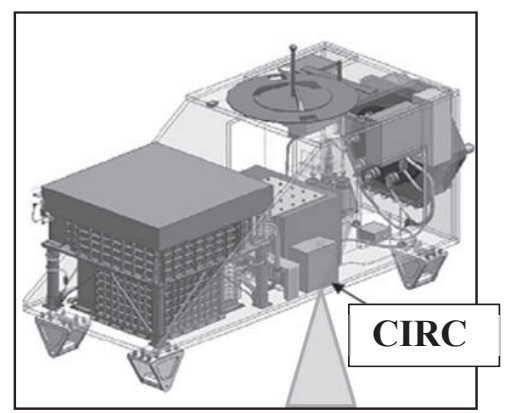

(c)

Fig. 1. Schematic view of (a) ALOS-2, (b) CALET, and (c) mounting location of CIRC.

size, light mass, and low power consumption. Moreover, the CIRC design is based on a commercial infrared camera and it employs commercial-off-the-shelf (COTS) components to reduce cost and time for development. It is also possible to increase the observational frequency of wildfires if CIRCs are carried on various satellites, which can exploit the camera's abovementioned key features.

\subsection{Specifications of CIRC}

The baseline specifications of the CIRC are listed in Table 2. We set the baseline specifications such as spatial resolution and dynamic range to meet the requirements for wildfire detection. ${ }^{(10)}$ The detector has a large format $(640 \times 480$ pixels $)$ to obtain a wide field of view. Spatial resolution is an important factor for wildfire detection. The spatial resolution is $200 \mathrm{~m}$ from an altitude of $600 \mathrm{~km}$ (ALOS-2) and $130 \mathrm{~m}$ from an altitude of $400 \mathrm{~km}$ (CALET). Eliminating the cooling system reduces the size $(110 \times 180 \times 230$ $\left.\mathrm{mm}^{3}\right)$ and electrical power consumption $(<20 \mathrm{~W})$. The CIRC PFMs on board the ALOS2 and CALET are shown in Fig. 2. The performance and functionality are not much different between both CIRCs. The CIRC is based on a commercial infrared camera. We modified the hardware design for purposes of space applications. CIRCs possess key technologies that are advantageous to space applications, i.e., microbolometer, athermal optics, and a shutterless system for achieving small size, reduced mass, and low power consumption. 
Table 2

Specifications of CIRC.

\begin{tabular}{lc}
\hline Parameter & Specifications \\
\hline Size & $110 \times 180 \times 230 \mathrm{~mm}^{3}$ \\
Mass & $3 \mathrm{~kg}$ \\
Detector & Uncooled infrared detector \\
Wavelength & $8-12 \mu \mathrm{m}$ \\
Number of pixels & $640 \times 480$ \\
Spatial resolution & $<200 \mathrm{~m} @ 600 \mathrm{~km}($ ALOS-2) \\
& $<130 \mathrm{~m} @ 400 \mathrm{~km}(\mathrm{CALET})$ \\
Field of view & $12^{\circ} \times 9^{\circ}$ \\
Exposure & $33 \mathrm{~ms}$ \\
Dynamic range & $180-400 \mathrm{~K}$ \\
Bit number & $14 \mathrm{bits}$ \\
NEDT & $0.2 \mathrm{~K} @ 300 \mathrm{~K}$ \\
FPN & $0.3 \mathrm{~K} @ 300 \mathrm{~K}$ \\
Temperature accuracy & $4 \mathrm{~K}(\mathrm{goal}: 2 \mathrm{~K} @ 300 \mathrm{~K})$ \\
\hline
\end{tabular}

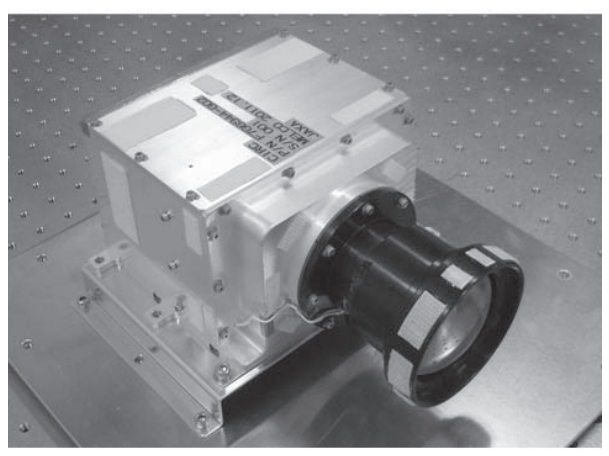

(a)

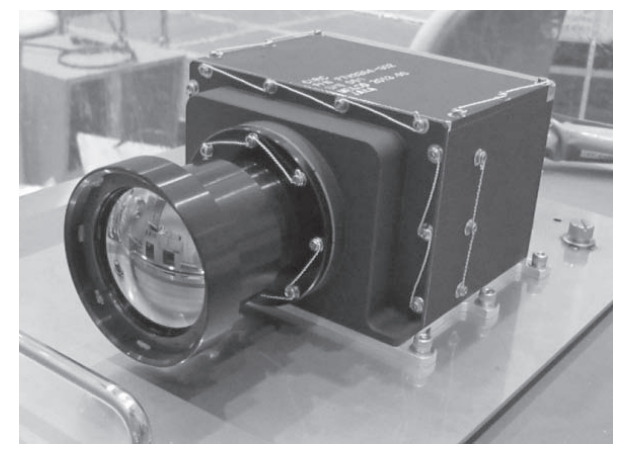

(b)

Fig. 2. CIRC PFM on board (a) ALOS-2 and (b) CALET.

\subsection{Microbolometer}

We adopted the use of microbolometers along the infrared (IR) focal plane array (FPA) of the CIRC. Microbolometers are based on the principle of detecting infrared energy as minute changes in the IR absorber temperature when an infrared photon is incident on the device. Their advantage is that they do not require a cooling system such as a mechanical cooler. 
The CIRC has a $640 \times 480$ pixel silicon-on-insulator (SOI) diode uncooled IR FPA developed by Mitsubishi Electric Corporation (MELCO), ${ }^{(1)}$ and is the largest microbolometer ever used for observations of Earth from space. Its pixel size is $25 \times 25$ $\mu \mathrm{m}^{2}$. The noise equivalent differential temperature (NEDT) is $40 \mathrm{mK}$ with $\mathrm{f} / 1$ optics. The drive and readout circuits are nearly identical to those of a commercial IR camera. For purposes of space application, we performed radiation damage tests and screening of commercial devices.

\subsection{Athermal optics}

We employ athermal optics to keep the optical performance in a wide range of temperature. This is an advantage of the CIRC given the restricted resources of satellites, because CIRC does not require active thermal control or a focus mechanism for the optics. The optics used in the CIRC consist of $\mathrm{f} / 1.2$ refractive optics. The focal length of the optics setup is $78 \mathrm{~mm}$. The temperature of the optics system of the CIRC changes in orbit. The temperature changes in the optics can lead to defocusing because the refractive indices of the lens materials are highly dependent on temperature. To compensate for this defocus, it is necessary to employ a focus mechanism or a heater to keep the optics temperature constant. However, such mechanisms increase the amount of sensor resources such as size, mass, and electric power consumption. Athermal optics can compensate for the defocus owing to temperature variation without the use of such mechanisms.

The optical design of the CIRC is shown in Fig. 3. The athermal optics of the CIRC compensate for the defocus by utilizing a combination of different lens materials and diffractive lenses. The optics of the CIRC use a germanium and a chalcogenide glass $\left(\right.$ GASIR $\left.^{\circledR} 1\right){ }^{(12)}$ Figure 4 shows the design value of the modulation transfer function (MTF) of the CIRC optics with respect to the ambient temperature range from -15 to $50{ }^{\circ} \mathrm{C}$. The designed MTF is constant over a wide range of temperatures.

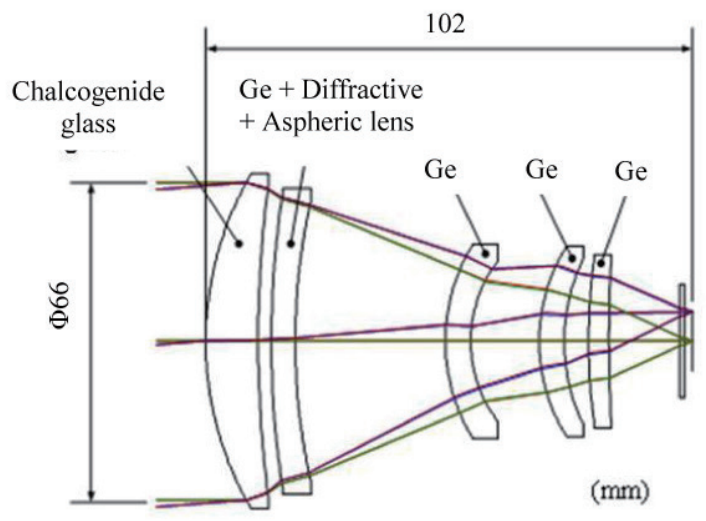

Fig. 3. (Color online) Optical design of CIRC. 


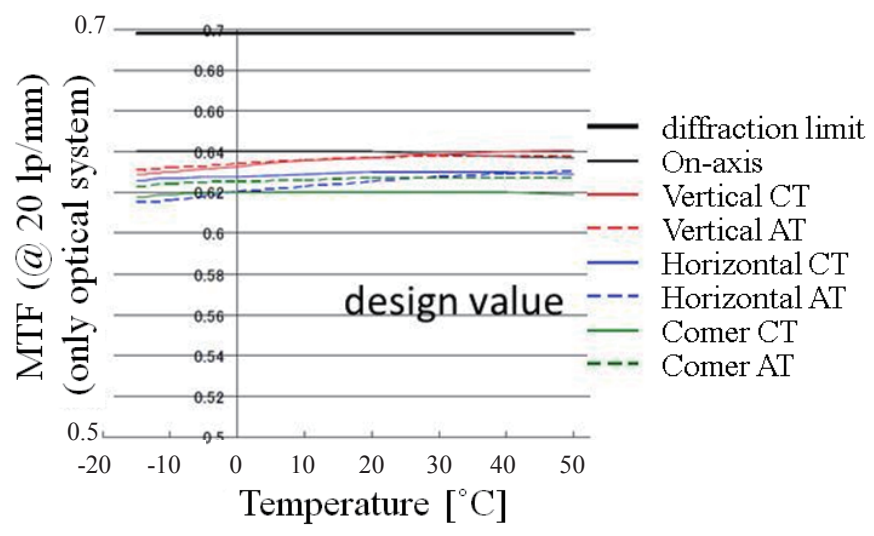

Fig. 4. (Color online) Calculated MTF of CIRC optics in the ambient temperature range from -15 to $50^{\circ} \mathrm{C}$. These MTF values indicate the design values of only the optical system. Line styles represent results at various positions and MTF directions.

\subsection{Shutterless system}

We eliminated the mechanical shutter from the CIRC, and this enabled us to downsize the CIRC. A mechanical shutter is more commonly used as a calibration source. Therefore, we devised a method of achieving temperature calibration and straylight correction from within the CIRC. We obtained images of a blackbody at various temperatures for different CIRC temperatures to perform stray-light correction using the temperature of the CIRC. The details are presented in $\S 4$.

\section{Ground Calibration Test of CIRC PFM On Board ALOS-2 and CALET}

We carried out a ground calibration test of the CIRC PFMs on board the ALOS2 and CALET at the Tsukuba Space Center at JAXA. CIRCs do not have a calibration function, which is challenging unlike in the case of previous space sensors. Therefore, we need to construct correction algorithm using only calibration data during the ground calibration test and CIRC's temperatures. The objectives of this calibration are (i) to construct a data correction algorithm and (ii) to confirm the imaging quality and radiometric quality of the CIRC with the calibrated images.

\subsection{Construction of data correction algorithm}

The stray light (i.e., the overall radiation except that from the target) had to be filtered out, because a background image cannot be taken without a calibration function. The stray-light intensity changes depending on the temperature of the CIRC. Therefore, we generated a calibration data table (stray-light and gain correction coefficients) and algorithm using blackbody images at various temperatures $\left(-30\right.$ to $\left.50^{\circ} \mathrm{C}\right)$ obtained with the CIRC PFM at various environmental temperatures $\left(-15\right.$ to $\left.50{ }^{\circ} \mathrm{C}\right)$. The flowchart 
used for the data correction is shown in Fig. 5, and each process in the flowchart is described below.

(I) Bad pixel correction is required to correct the brightness of a bad pixel by substituting the mean brightness of the normal surrounding pixels. A "bad" pixel is defined as a pixel that has more sensitivity than $\pm 19 \%$ of the average value of surrounding $11 \times 11$ pixels after estimation of sensitivity using blackbody images at 15 and $40{ }^{\circ} \mathrm{C}$. Pixels whose sensitivity changes within $30 \mathrm{~s}$ beyond the abovedefined value are also included as bad pixels.

(II) Dummy correction is carried out to subtract the electrical background noise using dummy pixels, namely, optical black pixels. Dummy pixels of the CIRC do not include a thermally insulating structure and an infrared ray absorbing structure. These pixels exhibit no sensitivity to incident infrared rays, and have electrical noise the same as effective pixels. Dummy correction technique is widely used in CMOS and CCD, and the optical black signal is subtracted from such an image signal to generate a corrected image signal. ${ }^{(13)}$ The CIRC has twenty dummy pixels per line. In the case of the CIRC, eighteen of them are used in this correction, and the average is subtracted from each pixel in the line.

(III) Stray-light correction is performed for each pixel using the stray-light correction coefficients, which represent the amount of stray light change with changes in the CIRC temperature. The procedure for calculating this coefficient is described later.

(IV) Gain correction represents the sensitivity correction performed for each pixel using the gain correction coefficients. By means of this gain correction, data of the CIRC are simultaneously converted from the digital number (DN) to the equivalent radiance or temperature.

Next, we describe the methods for stray-light and gain correction. These coefficients are calculated on the basis of the data table generated via the ground calibration test, which is described in $\S 4.2 .2$. The stray-light correction coefficient $(\beta)$ is defined using

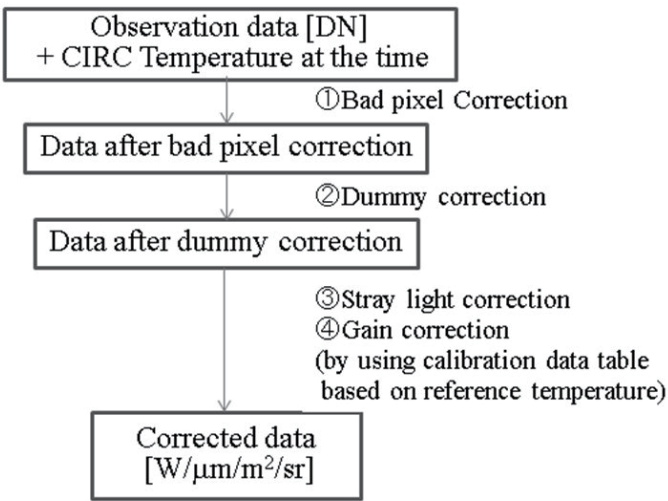

Fig. 5. Flow chart indicating procedure for data correction. 
eq. (1). This coefficient is measured in units of DN per $\mathrm{K}$, and it has the dimension of a $640 \times 480$ array.

$$
\beta=\frac{\text { Image } B-\operatorname{ImageA}}{T_{\text {ref_b }}-T_{\text {ref_a }}}
$$

Then, Image $A$ and Image $B$ represent the corrected data obtained after dummy correction with the same blackbody temperatures $(T b b)$ and different environmental temperatures (Fig. 6). The parameters $T_{\text {ref a }}$ and $T_{\text {ref b }}$ denote reference temperatures that correspond to the average of the lens and detector temperature of the CIRC under the tentative assumption that these temperatures mainly equally contribute to the stray light. The stray-light correction coefficients, depending on the reference temperature, indicate the amount of stray-light change with changes in the temperature of the CIRC. If the reference temperature of the CIRC is changed by $\Delta T$, the corresponding increase in stray light is expressed as $\beta \times \Delta T$.

Next, we calculate the gain correction coefficient $(\alpha)$. Data for calibration were obtained at different blackbody temperatures $\left(-30,-10,10,30\right.$, and $\left.50{ }^{\circ} \mathrm{C}\right)$. The first step in the calculation is to carry out stray-light correction for the calibration data to correspond to the reference temperature $\left(T_{\text {ref sd }}\right)$ of one observational data set. It results in the uniformity of stray light. Five data sets corrected using the above procedure for various blackbody temperatures $\left(-30,-10,10,30\right.$, and $\left.50{ }^{\circ} \mathrm{C}\right)$ are obtained in this manner. The gain correction coefficient is derived using the five data sets, and it is measured in units of $\mathrm{W} / \mu \mathrm{m} / \mathrm{m}^{2} / \mathrm{sr} / \mathrm{DN}$, as seen from eq. (2).

$$
\alpha=\frac{\left(\operatorname{Rad}_{T b b_{-} \mathrm{c}}\right)-\left(\operatorname{Rad}_{T b b_{-} \mathrm{d}}\right)}{\text { ImageC }- \text { ImageD }_{\text {mag }}}
$$

The parameters $\operatorname{Rad}_{T b b_{\mathrm{C}} \mathrm{c}}$ and $\operatorname{Rad}_{T b b_{\mathrm{d}}}$ denote the radiances corresponding to each blackbody temperature (Fig. 7). ImageC and ImageD denote two of the five data sets with different blackbody temperatures. Four gain correction coefficients ( $\alpha 1$ to $\alpha 4)$ are obtained every $20{ }^{\circ} \mathrm{C}$ (Fig. 8). The gain correction coefficients relate the radiance and DN. An observational data set $\left(D N_{\mathrm{obs}}\right)$ is calibrated using the appropriate gain coefficients using eq. (3),
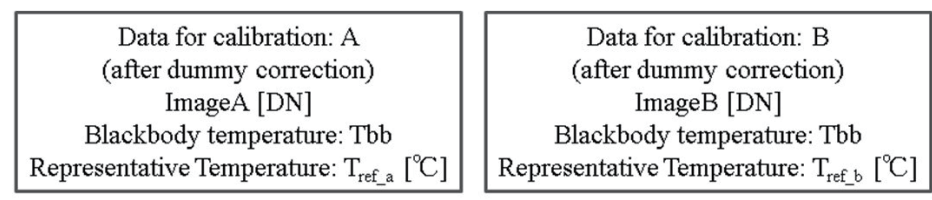

Fig. 6. Assumed parameters of images A and B. 


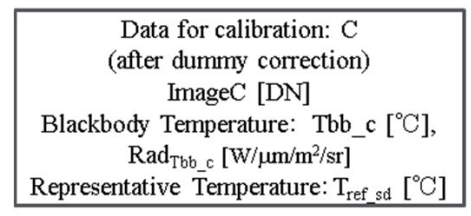

Fig. 7. Assumed parameters of images $\mathrm{C}$ and $\mathrm{D}$.

\section{Gain Correction Coefficient $(\alpha)$}

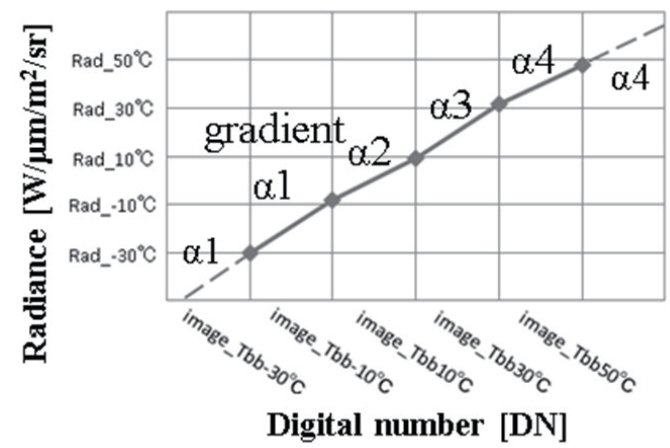

Fig. 8. Four gain correction coefficients corresponding to a set of reference temperatures.

$$
\operatorname{Rad}_{\mathrm{obs}}=\operatorname{Rad}_{T b b}+\alpha \times\left(D N_{\mathrm{obs}}-D N_{T b b}\right),
$$

where $D N_{\text {obs }}$ is equal to the $\mathrm{DN}$ in the case of $D N_{T b b}<D N_{\text {obs }}<D N_{T b b+20}$. These $D N_{T b b}$ and $D N_{T b b+20}$ values indicate the DN at the blackbody temperatures of $T b b$ and $T b b+20{ }^{\circ} \mathrm{C}$, respectively. The radiance at $T b b$ is $\operatorname{Rad}_{T b b}$. $\operatorname{Rad}_{\mathrm{obs}}$ denotes the data converted to radiance from $D N_{\text {obs }}$. In this manner, the observed data can be converted to the corresponding values of radiance.

\subsection{Performances}

\subsubsection{Imaging quality}

The MTF was measured to evaluate the imaging quality of the CIRC at various operating temperatures $\left(-15\right.$ to $\left.50{ }^{\circ} \mathrm{C}\right)$. The experimental setup for MTF measurement is shown in Fig. 9. Collimated infrared rays were made to pass through a germanium window located on the side of the vacuum chamber. The CIRC was able to capture images of a four-bar target. Figure 10 shows the measurement points of the four-bar target for MTF, at the center, $\pm 5^{\circ}$ along the cross-track (CT) direction, and $\pm 3.5^{\circ}$ along the along-track (AT) direction. 

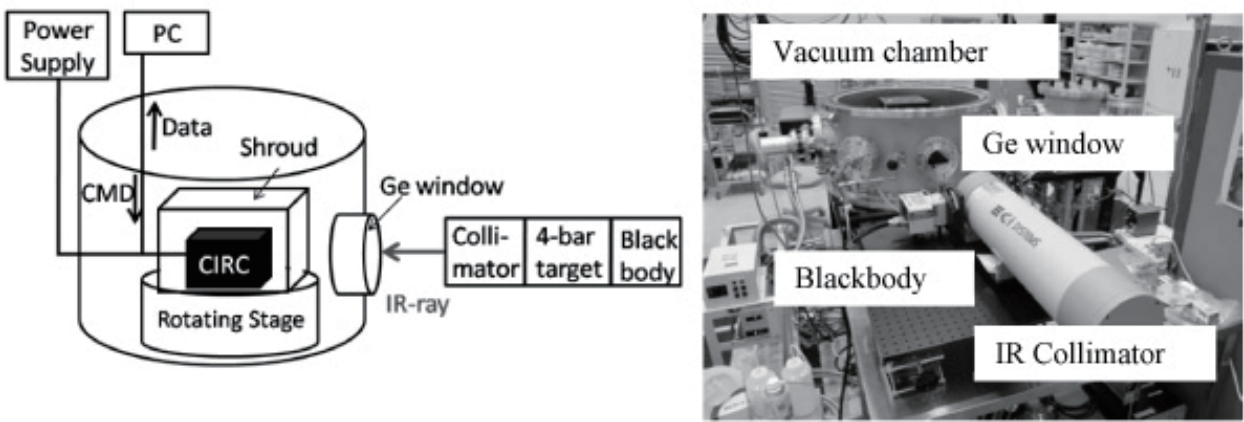

Fig. 9. Experimental setup of MTF measurement.

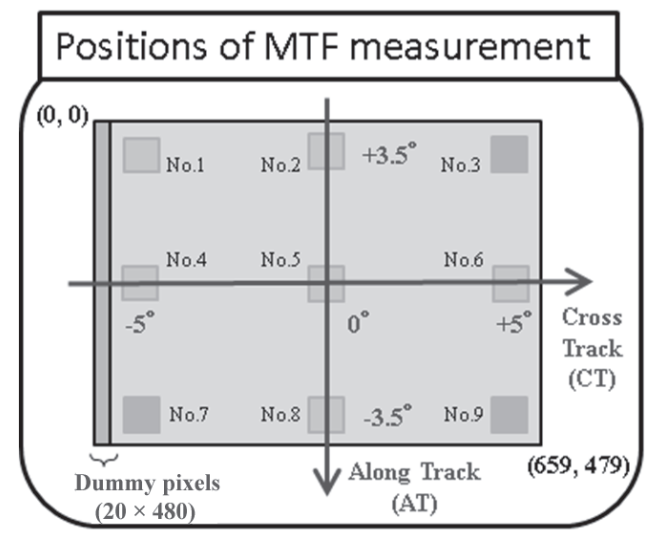

Fig. 10. Measurement points of MTF on image acquired by CIRC. The nine positions are marked Nos. 1 to 9 . Dummy pixels $(20 \times 480)$ are optical black pixels, which exhibit non-photosensitivity and have the same electrical properties as standard effective pixels.

Figure 11 shows the MTF results of the CIRC PFMs for the ALOS-2 and CALET. The MTF along the CT direction for the ALOS-2 [Fig. 11(a)] is constant with a value in the range from 0.2 to 0.39 , which is independent of environmental temperature. In contrast, the MTF along the AT direction [Fig. 11(b)] is slightly lower in low-temperature environments. In the case of the CIRC PFM for the CALET, the MTF values range between 0.24 and 0.33 along the CT direction [Fig. 11(c)] and from 0.34 to 0.54 along the AT direction [Fig. 11(d)]. The trend of the environmental temperature dependence is the same as that observed in the results of the CIRC PFM for ALOS-2. As a result, the MTF of the CIRC was found to be effective in capturing clear images across the entire range of operating temperatures for both PFMs. Thus, we confirmed the athermal optical performance of the CIRC. 


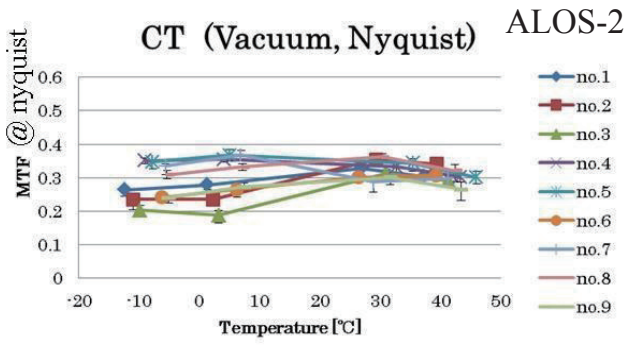

(a)

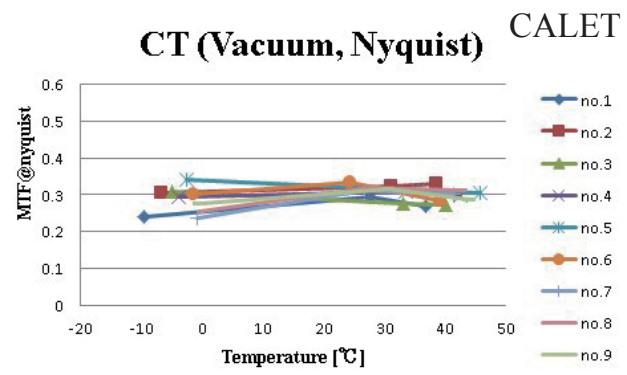

(c)

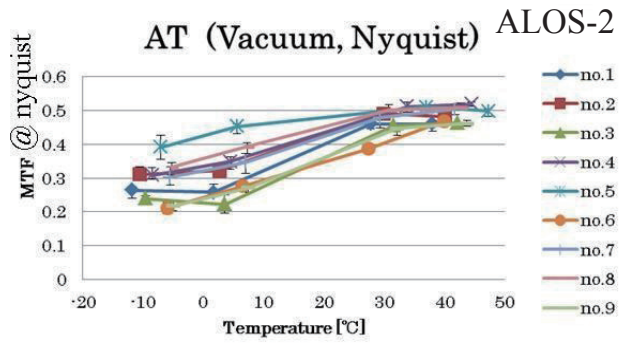

(b)

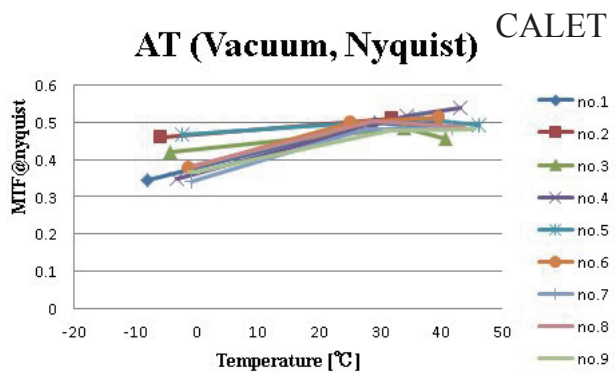

(d)

Fig. 11. (Color online) Measurement results of MTF on board ALOS-2 [(a) and (b)] and CALET [(c) and (d)]. (a) and (c) display MTF values along the CT direction, while (b) and (d) represent those along the AT direction.

\subsubsection{Radiometric quality}

The experimental setup for generating the calibration data table is shown in Fig. 12. The CIRC was installed in a vacuum chamber and enclosed by a shroud to control its ambient temperature with a heater and cooler. The blackbody was set up in the front of the lens. Figure 13 depicts the data table of the blackbody temperatures ( -30 to 50 ${ }^{\circ} \mathrm{C}$, every $20^{\circ} \mathrm{C}$ ) obtained with the CIRC PFM at various environmental temperatures $\left(-15\right.$ to $50{ }^{\circ} \mathrm{C}$, every $1{ }^{\circ} \mathrm{C}$ ). Radiometric calibration is performed by interpolating the coefficients within the range of the data table.

The NEDT and fixed pattern noise (FPN) values were less than 0.2 and $0.3 \mathrm{~K}$, respectively (Table 3 ). These results are consistent with the design values of the CIRC, and they satisfy its specifications. Figure 14 shows a comparison between a raw image and a corrected image obtained using the CIRC PFM for the ALOS-2. Performing data correction makes it possible to capture smooth images without a calibration function. The temperature accuracy is less than $2 \mathrm{~K}$ upon a comparison of the brightness temperature of the corrected image and the actual blackbody temperature. This is within the specification for the temperature correction accuracy. In the case of the CIRC PFM for the CALET, the temperature accuracy is from 0.5 to $2.8 \mathrm{~K}$, which is also within the specification. 


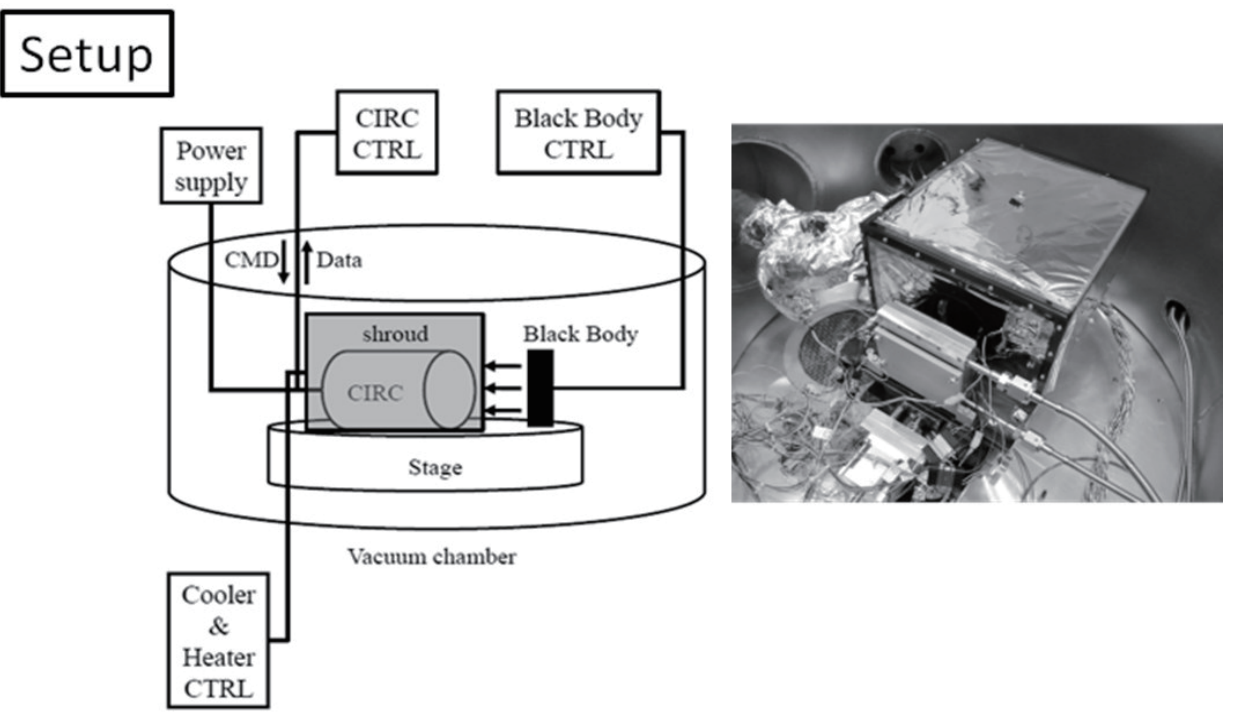

Fig. 12. Experimental setup for generating calibration data table.

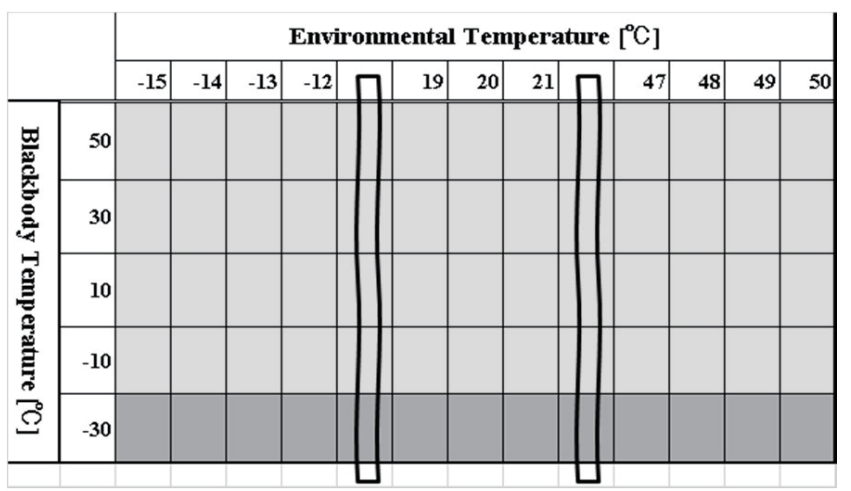

Fig. 13. Calibration data table for radiometric correction. The data at various blackbody temperatures $\left(-30\right.$ to $50^{\circ} \mathrm{C}$, every $\left.20^{\circ} \mathrm{C}\right)$ were obtained at various environmental temperatures $(-15$ to $50^{\circ} \mathrm{C}$, every $\left.1{ }^{\circ} \mathrm{C}\right)$.

Table 3

Results of NEDT and FPN.

\begin{tabular}{lcc}
\hline & NEDT (K) & FPN (K) \\
\hline for ALOS-2 & 0.19 & 0.27 \\
for CALET & 0.18 & 0.21 \\
\hline
\end{tabular}




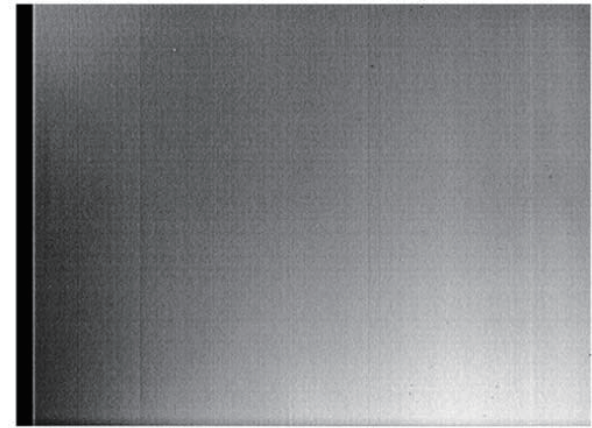

(a)

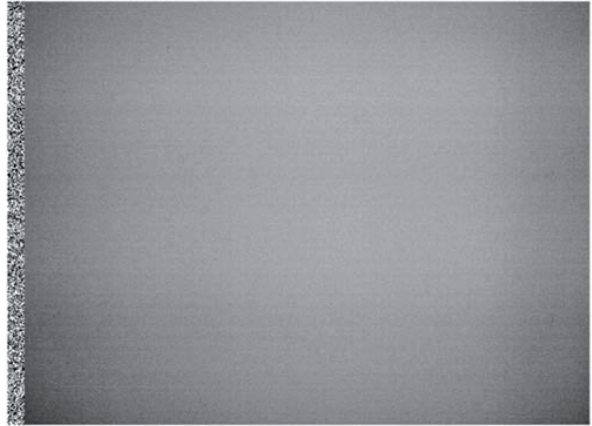

(b)

Fig. 14. Blackbody image obtained by CIRC on board ALOS-2. (a) and (b) show the raw image and corrected image, respectively.

\section{Airborne Observation with CIRC Ground Test Model}

We carried out airborne observations using the ground test model (GTM) of the CIRC, which is a roughly similar to the PFM and has optical and radiometric performances equivalent to the corresponding PFM. The CIRC GTM will be used for vicarious calibration to compare with on-board data of the PFM. Airborne observation is one of the vicarious calibration experiments, and it is thought to be necessary because of the CIRC without a calibration function. This section shows results of first airborne experiments using the CIRC GTM to check the data correction algorithm as described in $\S 4$.

Observational flights were carried out on March 22 and 28, 2012. The aircraft used was a Cessna172 Skyhawk made by the Cessna Aircraft Company. The observation area was Tsukuba City, which is south of Ibaraki Prefecture and Narita City in Chiba Prefecture, Japan. The flight altitude ranged from 300 to $750 \mathrm{~m}$. The ground sample distance (GSD) at these altitudes ranged from about 10 to $25 \mathrm{~cm}$.

Figure 15 shows an aerial image of the Tsukuba Space Center, which is one of the facilities in JAXA, captured from an altitude of $750 \mathrm{~m}$ with a compact digital camera. The yellow rectangle represents the field of view of the CIRC. Figure 16 shows a comparison between the raw image and corrected image captured by an airborne observation from an altitude of $750 \mathrm{~m}$. We confirmed that the data were corrected without a calibration function and that the actual images could be corrected using the ground calibration data.

The temperature correction accuracy was verified using the image of the pond area shown in Fig. 16(b), indicated by the white box. The temperature of the pond measured using a water temperature gauge was about $11^{\circ} \mathrm{C}$. The corrected data obtained using the CIRC GTM yielded a temperature of $11.5^{\circ} \mathrm{C}$, with the assumption that the emissivity of water was 0.98 . The temperature correction accuracy was about $0.5^{\circ} \mathrm{C}$, which is within the specification of $2 \mathrm{~K}$ for an environmental temperature of $\sim 300 \mathrm{~K}$. 


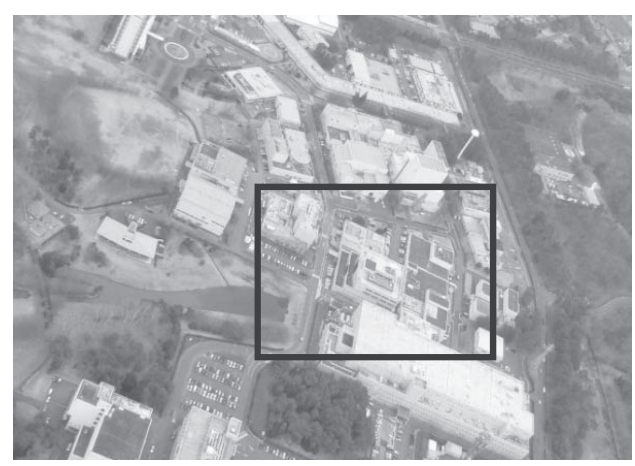

Fig. 15. Aerial image of Tsukuba Space Center from an altitude of $750 \mathrm{~m}$.

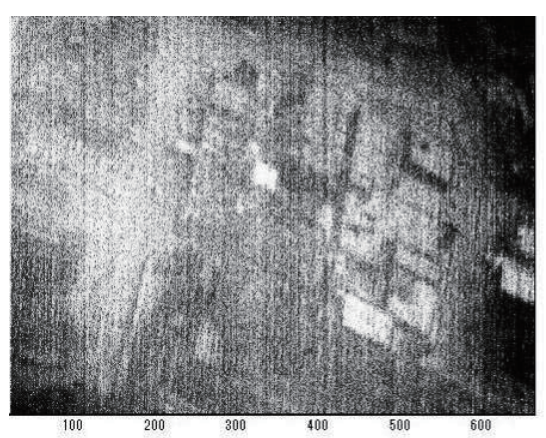

(a)

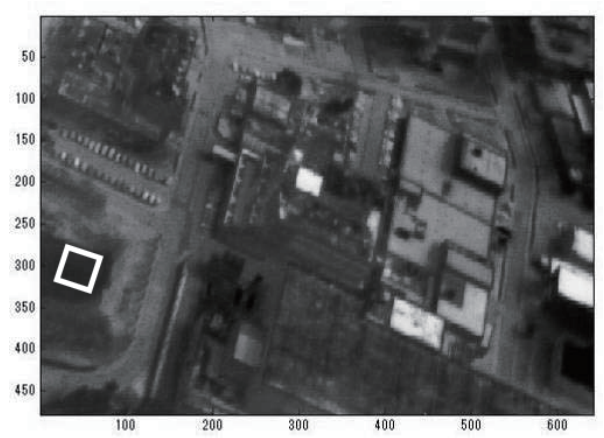

(b)

Fig. 16. Images captured by airborne observation: (a) raw image and (b) corrected image in which the white box indicates the pond area.

\section{Conclusions}

The CIRC is the first microbolometer sensor without a calibration function for space application, and achieves a small size, light mass, and low electrical power consumption by employing athermal optics and a shutterless system. We accomplished the method of temperature correction to achieve the temperature accuracy of $4 \mathrm{~K}$ without a calibration function in orbit. We have also confirmed that other performances (MTF and NEDT) are satisfied, and these devices are launch-ready. In addition, the data obtained through airborne observations could be corrected by the method of temperature correction, and the temperature correction accuracy was within the specification of $4 \mathrm{~K}$. This study represents an important first step in the application of a microbolometer for Earth observations. 


\section{Acknowledgements}

The authors would like to thank the CIRC team members in this work.

\section{References}

1 T. Fukuhara, M. Taguchi, T. Imamura, M. Nakamura, N. Iwagami, M. Ueno, M. Suzuki, G. L. Hashimoto, M. Sato, A. Yamazaki, R. Kashikawa, I. Higashino and K. Noguchi: Proc. SPIE 6965 (2008) 694030.

2 J. D. Spinhirne, V. S. Stanley, J. Cavanaugh, S. Palm, K. Manizade, J. W. Hoffman and R. C. Grush: Proc. SPIE 3379 (1998) pp. 14-21.

3 D. M. Winker, J. Pelon, and M. P. McCormick: Proc. SPIE 4893 (2003) pp. 1-11.

4 ESA: EarthCare-Earth Clouds, Aerosols and Radiation Explorer Technical and Programmatic Annex, http://esamultimedia.esa.int/docs/EEUCM/EarthCARE_TPA.pdf (accessed in April 2004).

5 H. Katayama, M. Naitoh, M. Suganuma, M. Harada, Y. Okamura, Y. Tange and K. Nakau: Proc. SPIE 7458 (2009) 745806.

6 E. Kato, H. Katayama, M. Naitoh, M. Harada, R. Nakamura and R. Sato: Proc. SPIE 8353 (2012) $8353 \mathrm{~J} 1$.

7 M. Naitoh, H. Katayama, M. Harada, R. Nakamura, E. Kato, Y. Tange, R. Sato and K. Nakau: Proc. ICSO ICSO-066 (2012).

8 S. Suzuki, Y. Osawa, Y. Hatooka, Y. Kankaku and T. Watanabe: Proc. SPIE 7474 (2009) 74740Q.

9 S. Torii, M. Hareyama, N. Hasebe, K. Kasahara, S. Kobayashi, D. Kodaira, H. Murakami, S. Ozawa, S. Udo, N. Yamashita, K. Ebisawa, H. Fuke, J. Nishimura, Y. Saito, M. Takayanagi, H. Tomida, S. Ueno, T. Yamagami, K. Hibino, S. Okuno, T. Tamura, N. Tateyama, T. Kobayashi, T. Kotani, K. Yamaoka, A. Yoshida, Y. Shimizu, M. Takita, T. Yuda, Y. Katayose, M. Shibata, E. Kamioka, A. Kubota, K. Yoshida, M. Ichimura, S. Kuramata, Y. Tunesada, T. Terasawa, H. Kitamura, Y. Uchihori, Y. Komori, K. Mizutani, K. Munakata, A. Shiomi, J. W. Mitchell, A. J. Ericsson, T. Hams, J. F. Krizmanic, A. A. Moissev, M. Sasaki, J. F. Ormes, M. L. Cherry, T. G. Guzik, J. P. Wefel, W. R. Binns, M. H. Israel, H. Krawczynski, P. S. Marrocchesi, M. G. Gagliesi, G. Bigongiari, A. Caldarone, M. Y. Kim, R. Cecchi, P. Maestro, V. Millucci, R. Zei, C. Avanzini, T. Lotadze, A. Messineo, F. Morsani, O. Adirani, L. Bonechi, P. Papini, E. Vannuccini, J. Chan, W. Gan, T. Lu, Y. Ma, H. Wang and G. Chen: Proc. SPIE 7021 (2008) 702114 .

10 H. Katayama, Y. Okamura, Y. Tange and K. Nakau: Proc. ISTS n-30p (2008).

11 M. Ueno, Y. Kosasayama, T. Sugino, Y. Nakaki, Y. Fujii, H. Inoue, K. Kama, T. Seto, M. Takeda and M. Kimata: Proc. SPIE 5783 (2005) p. 566.

12 Umicore, Electro-Optic Materials: GASIR ${ }^{\circledR} 1$, http://optics.org/buyers/company/C000001606/ gasir1.

13 J. C. Ahn and S. Si: United States Patent US7839438 (2010). 